\title{
Et si les ethnosciences facilitaient la production de passerelles au sein du monde académique comme non-académique?
}

What if ethnoscience helped building bridges within the academic and non-

academic world?

\section{Catherine Sabinot}

\section{(2) OpenEdition}

Journals

Édition électronique

URL : https://journals.openedition.org/ethnoecologie/8500

DOI : $10.4000 /$ ethnoecologie.8500

ISSN : 2267-2419

Éditeur

Laboratoire Éco-anthropologie

Référence électronique

Catherine Sabinot, «Et si les ethnosciences facilitaient la production de passerelles au sein du monde académique comme non-académique ? », Revue d'ethnoécologie [En ligne], 20 | 2021, mis en ligne le 31 décembre 2021, consulté le 06 mars 2022. URL : http://journals.openedition.org/ethnoecologie/8500 ; DOI : https://doi.org/10.4000/ethnoecologie.8500

Ce document a été généré automatiquement le 6 mars 2022.

Revue d'ethnoécologie est mis à disposition selon les termes de la licence Creative Commons Attribution - Pas d'Utilisation Commerciale - Pas de Modification 4.0 International. 


\section{Et si les ethnosciences facilitaient la production de passerelles au sein $\mathrm{du}$ monde académique comme non- académique?}

What if ethnoscience helped building bridges within the academic and nonacademic world?

Catherine Sabinot

\section{La Salle Auguste Chevalier, un passage inspirant vers l'ethnoécologie d'aujourd'hui}

1 C'est un très grand honneur pour moi d'avoir été sollicitée par les éditeurs de la Revue d'ethnoécologie pour ce numéro anniversaire, et d'être ainsi comptée, avec beaucoup d'humilité, parmi celles et ceux qui s'inscrivent dans le "panorama d'un siècle d'histoire de l'étude des relations entre les sociétés et leur environnement ». Cela fait à peine vingt ans que j'ai poussé la porte de la Salle Auguste Chevalier au Jardin des Plantes, où j'ai eu la chance d'apprendre, de débattre et de me nourrir de multiples manières de voir le monde, de le penser et de le vivre. Ces riches moments se déroulaient tant durant les sessions d'enseignements du diplôme d'études approfondies (DEA) pour lequel j'avais rejoint le Muséum national d'Histoire naturelle de Paris que pendant les temps de partage autour de curiosités culinaires, solides ou liquides, ramenées par l'un ou l'autre des étudiants ou chercheurs impatients de les faire découvrir aux collègues et amis.

2 Cette salle riche de souvenirs se nommait la salle Auguste Chevalier, et bien qu'il s'agissait d'une des salles d'un préfabriqué - car de longs travaux avaient été engagés sur les bâtiments anciens -, elle portait en elle beaucoup d'Histoire et d'histoires. Elle était habitée de nombreux objets provenant de divers endroits du monde et 
d'étonnants récipients de verre abritant des espèces inattendues. Les ruches de toutes sortes faites d'éléments végétaux étaient remarquables et faisaient vivre les lieux: savoirs, savoir-faire et modes de pensées variés y vibraient. Auguste Chevalier, spécialiste d'agronomie tropicale et "pré-ethnoécologue» de son époque (l'ethnoécologie ainsi nommée n'existait pas encore) m'était alors inconnu, tout comme la plupart des chercheurs du $\mathrm{xx}^{\mathrm{e}}$ siècle impliqués dans l'étude des relations au vivant dans leurs diverses dimensions: décrire et inventorier les éléments de la nature, recenser des manières de nommer les plantes, les animaux, les minéraux, les artéfacts dans les langues locales, décrire et analyser l'utilisation que les populations de chaque localité en font, puis étudier les normes d'interactions propres à chaque société, les normes de gestion de l'environnement, des environnements, des natures... Les racines de mon attachement à comprendre les liens entre les humains et les non-humains, ainsi que les interactions entre humains comme je le fais aujourd'hui en tant que chercheure à l'Institut de Recherche pour le Développement (IRD), ont été particulièrement nourries par ces chercheurs et professeurs du Muséum, marquées par des personnalités dont le savoir avait à un moment grandi ou traversé le Jardin des plantes. Parmi ces personnalités aujourd'hui disparues, il y a Auguste Chevalier qui a entre autres lancé en 1921 cette revue originale devenue aujourd'hui la Revue d'ethnoécologie (en ligne) qui permet de rassembler une diversité de publications sur les relations entre les sociétés et leurs environnements. Il y aussi Jacques Barrau, André-Georges Haudricourt, Claudine Friedberg qui nous a récemment quitté et encore Théodore Monod et Edgar Morin, qui bien que ne s'ancrant pas dans le champ disciplinaire de l'ethnoécologie, ont profondément contribué par leur humanisme et leurs approches transversales du monde marin comme désertique pour le premier, et des sociétés dans toute leur complexité pour le second, à guider nos apprentissages et nos recherches dans les années 2000. Ces auteurs français ont marqué ces années passées au Muséum et continuent de nourrir mes travaux aujourd'hui.

Basée depuis 2013 au centre IRD de Nouméa en Nouvelle-Calédonie en tant qu'ethnoécologue étudiant les savoirs, savoir-faire et normes des sociétés insulaires et littorales, je travaille en interaction très étroite avec les institutions du territoire comme avec les institutions alentours des pays du Pacifique tels le Vanuatu ou Fidji. Les partenaires de recherche des Universités et des Instituts de recherche locaux sont précieux et ont tous été formés à d'autres disciplines que l'ethnoécologie. La plupart d'entre eux ne connaissaient pas ce champ disciplinaire. Ceux avec lesquels les partenariats sont les plus solides relèvent de la géographie, de l'écologie et des sciences du climat. Nous montrerons dans les pages qui suivent comment l'ethnoécologie a été pour nous au fondement de la création de ces passerelles entre les champs disciplinaires au travers de quelques programmes de recherche à visée fondamentale et appliquée. De plus, dans les petits territoires insulaires et archipélagiques du Pacifique, les rapports que les chercheurs peuvent entretenir avec les agents des services des collectivités publiques d'échelle locale, provinciale, gouvernementale ou étatique comme avec les responsables coutumiers sont particulièrement étroits et rendent non seulement possible un partage réel des résultats de nos recherches mais aussi une relativement bonne mobilisation de nos recherches par les acteurs produisant, ajustant et mettant en œuvre les politiques publiques. Nous verrons qu'étudier les relations entre sociétés et natures par le prisme de l'ethnoécologie a facilité l'implication des ethnoécologues et anthropologues de l'environnement dans l'analyse des défis majeurs de gestion des territoires du Pacifique, ainsi que dans la formation de jeunes dans ces 
approches. Enfin, nous conclurons sur l'engagement grandissant dans les organes intergouvernementaux des ethnoécologues et des chercheurs s'intéressant à l'étude des savoirs locaux.

4 Avant ces courts développements qui montreront comment les ethnosciences se sont montrées être un champ de compréhension du monde essentiel pour reconnaître et faire reconnaître les savoirs des praticiens de l'environnement, ceux qui ont des interactions régulières, voire quotidiennes avec les non-humains, permettez-moi de partager une petite histoire d'un chemin qui conduit à l'ethnoécologie, discipline en réalité bien peu connue en dehors de celles et ceux qui la pratiquent ou qui côtoient celles et ceux qui la pratiquent...

\section{Petite histoire d'un chemin qui conduit à l'ethnoécologie}

5 Alors que je terminais mes années lycée en 1998, j'ignorais l'existence de l'anthropologie et de l'ethnoécologie. Histoire, géographie, lettres et études des langues étrangères et des langues anciennes étaient les seules disciplines des sciences humaines et sociales que j'avais clairement identifiées. Ce qui était certain à mes yeux était que je souhaitais continuer d'apprendre, et d'apprendre à apprendre. J'avais envie de connaître, de comprendre, de questionner le monde. Ma seule évidence à l'époque était qu'il était possible de construire ce chemin en parcourant divers pays, en rencontrant des sociétés autres que celle qui nous avaient façonnées et en fréquentant les bancs de l'Université.

6 Connaître devait permettre de prendre soin : prendre soin de l'océan, de la terre, de ce qui nous lie à l'océan, au monde... Ce chemin devait aussi me nourrir pour me donner les moyens de transmettre l'envie d'apprendre aux plus jeunes, de leur donner le goût et les outils pour pouvoir confronter les points de vue, pour se constituer un esprit critique. Ignorant que les sciences humaines et sociales enseignées à l'Université pouvaient conduire à questionner les rapports entre les humains et leurs environnements, je choisis d'une part de saisir les opportunités de voyages et de rencontres, et d'autre part d'étudier la biologie, discipline qui à l'époque me semblait la plus proche des hommes dans le champ des sciences dites naturelles. Elle fut marine pour me rapprocher de l'espace océanique, des littoraux, ces territoires d'interface, ces lieux de rencontres, de mystère et aussi ces lieux d'attente. Je choisis Brest; ou Brest me choisit. Après ma licence, j'y découvris l'existence d'une maîtrise en gestion de l'environnement littoral pendant laquelle je reçus des enseignements en écologie, sociologie, géographie, économie, droit, biologie des pêches et je rencontrai des praticiens de la mer et de la gestion du littoral venant partager leur expérience. Mon chemin prenait peu à peu un cap plus humain, où les disciplines des sciences humaines et sociales se présentaient enfin comme essentielles et ajustées à mes envies et mes questionnements.

7 M'attacher à mieux comprendre les interactions entre les hommes et les milieux qu'ils habitent, pensent et vivent, deviendra mon cœur de métier. Après avoir suivi des enseignements au Muséum national d'Histoire naturelle de Paris où je découvrais véritablement les ethnosciences et tous ces «ethno-machins» (Friedberg 2005) résolument ancrés dans l'anthropologie, je me suis lancée dans une recherche 
doctorale sous la direction de Serge Bahuchet, directeur du Département Hommes, natures, sociétés au Muséum. Il coordonnait alors un programme de recherche s'intéressant à la dynamique des savoirs chez les sociétés forestières et me donna l'opportunité de m'y intégrer en étudiant les sociétés littorales, leurs savoirs, leurs pratiques et leurs manières d'être dans le monde, ce que j'ai continué de faire jusqu'à ce jour et que je ne projette pas de cesser (Figure 1). Cette thèse doctorale portait sur les transformations des savoirs, savoir-faire et savoir-être chez les pêcheurs du Gabon et sur l'évolution des modalités de transmission de ces savoirs (Sabinot 2008) (Figure 2). Elle s'est pleinement inscrite dans le champ de l'ethnoécologie, tout en se nourrissant d'auteurs relevant entre autres de l'anthropologie maritime, de l'anthropologie des pêches, de celles des techniques et des connaissances. Enfin, en rejoignant en 2009 le département d'anthropologie de l'Université Laval à Québec et en travaillant de manière très étroite avec Sabrina Doyon, professeure d'anthropologie au département, j'ai mesuré tout l'intérêt de nourrir nos réflexions d'ethnoécologues, d'anthropologues s'intéressant aux relations entre les sociétés et leurs environnements, à celles des penseurs de l'écologie politique (Doyon \& Sabinot 2015). J'ai eu la chance de pouvoir articuler, confronter et questionner ces approches disciplinaires sur des territoires et des pays des Océans atlantiques, indiens et pacifiques avec les chercheurs résidant ces lieux et contribuant chacun à consolider les outils et concepts susceptibles de nourrir nos réflexions.

Figure 1 : Des pêcheurs de Port-Gentil sur leur campement d'Ozouri. Les chants accompagnent les moments de ramendage

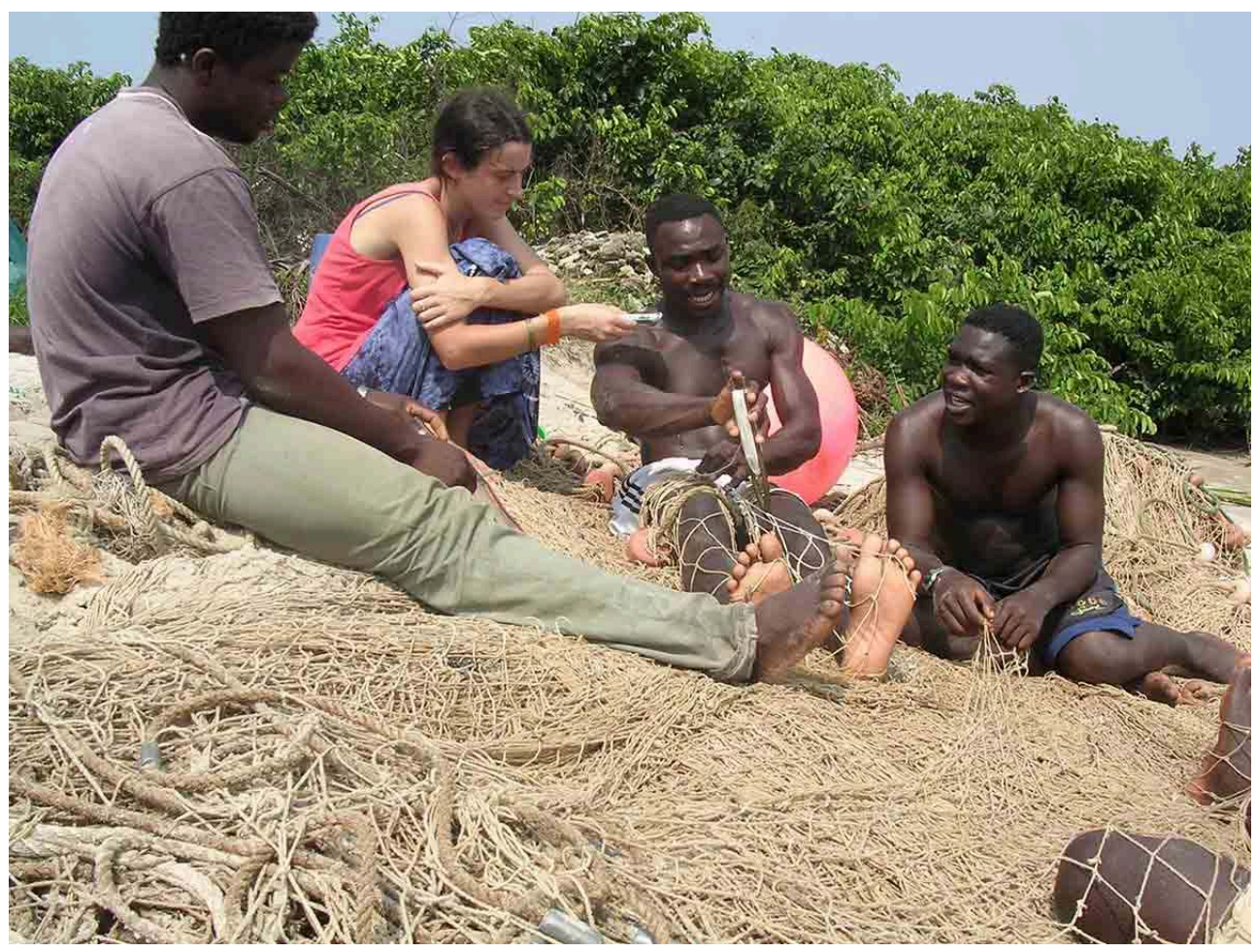

Collection C. Sabinot 
Figure 2 : Un jeune pêcheur montrant comment monter les plombs sur un filet à Louando au Gabon, 2006

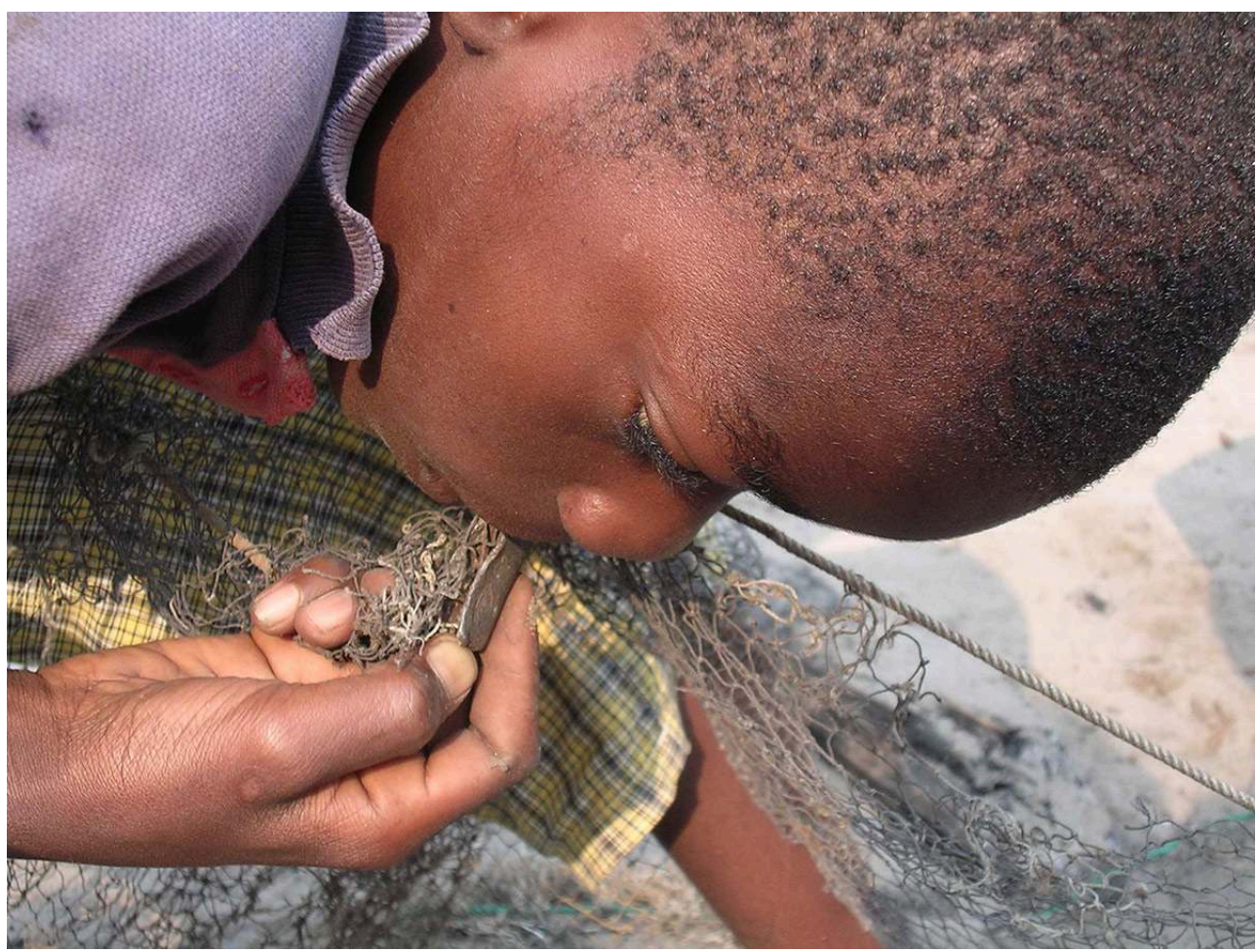

Photographie C. Sabinot

\section{L'ethnoécologie au cœur de recherches interdisciplinaires}

8 Aujourd'hui, les recherches que je mène dans l'Indopacifique portent sur les savoirs écologiques, sur les valeurs et les normes associées à ces savoirs et aux sociétés qui les construisent et les transforment au rythme des changements socio-économiques, politiques et environnementaux. Elles ne pourraient être si riches sans le concours des collègues des autres disciplines. En dialogue avec ces derniers relevant principalement des sciences naturelles, des sciences du climat, des sciences de l'éducation et de la géographie, ce sont l'ethnoécologie dans ces diverses dimensions et l'écologie politique qui me permettent de saisir, décrire et analyser les conséquences des modifications socio-environnementales sur les sociétés insulaires et littorales, leurs savoirs et leurs manières d'être au monde, ainsi que les enjeux que ces modifications sous-tendent pour l'adaptation des sociétés humaines à un monde qui change.

Les ethnosciences, durant mon expérience de chercheure sur les trois océans, sont devenues à la fois sujets de débats et leviers de coproductions avec le monde scientifique dans sa complexité et sa diversité; elles interviennent aussi avec les pêcheurs, les autres praticiens de la terre et de la mer et les acteurs de la conservation et du développement œuvrant dans les collectivités publiques, les associations ou les organisations non gouvernementales.

10 Avec le temps, les savoirs et manières de voir le monde révélés par ce champ de recherche ont traversé les frontières académiques et sont aujourd'hui fréquemment au 
cœur de programmes de recherche réellement interdisciplinaires. L'ethnoécologie, dans le champ de l'anthropologie, s'est pour nous exprimée comme une discipline facilitant l'interface et la création de passerelles. Parmi les projets de recherche structurants de cette interface dans le Pacifique, certains ont été financés par l'Union européenne ou l'Agence nationale de la recherche française, d'autres par les collectivités publiques provinciales ou encore par le Fonds Pacifique ou la Fondation de France. Les ambitions que suivaient les équipes de recherche pluridisciplinaires impliquées étaient d'une part, d'apporter quelques pierres aux avancées de la discipline de chacun ; d'autre part, il s'agissait de véritablement croiser les outils et méthodes des uns et des autres pour produire d'autres types de connaissances tentant d'offrir une perspective plus complète des dynamiques étudiées avec un potentiel de mobilisation accrue par les acteurs des politiques publiques.

Figure 3 : Entretien avec H. Vandegou, Îles des Pins, Nouvelle-Calédonie, 2016

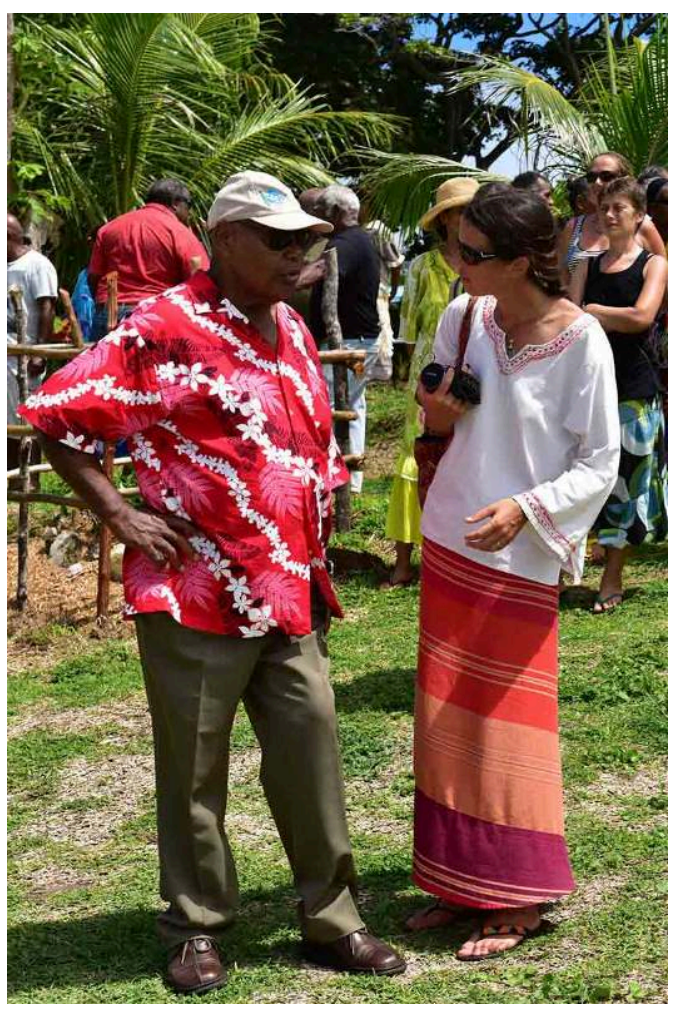

Photographie M. Juncker

C'est ainsi qu'en 2014 un collègue écologue de l'Institut agronomique néo-calédonien, Fabrice Brescia, spécialiste entre autres des populations de chauve-souris et de roussettes en Océanie, m'a sollicitée pour répondre à des questions de recherche se posant sur les îles Loyauté en Nouvelle-Calédonie: quelles sont les espèces de roussettes présentes? Comment se comportent-elles? Comment les populations ontelles évolué ? Mais aussi que représentent ces espèces pour les Kanak de l'archipel ? Comment sont-elles chassées et pourquoi ? Existe-t-il des règles de gestion ? ... et d'autres questions encore qu'il importait de résoudre avant que ne soit rédigé un code de l'environnement intégrant des textes sur les espèces protégées et sur la chasse. Des connaissances écologiques sur les populations de roussettes de la Grande Terre, île principale de la Nouvelle-Calédonie, mais elles étaient quasi inexistantes sur les îles 
Loyauté, quatre îles d'une superficie totale de près de $2000 \mathrm{~km}^{2}$ où vivent 18353 habitants (ISEE 2020). Alors que je menais jusque-là des recherches portant sur des problématiques liées à la mer, F. Brescia m'a convaincue par son ambition interdisciplinaire concrète de m'éloigner légèrement de mes thématiques habituellement océaniques et côtières (quoique... les roussettes ont des liens forts avec l'océan, considéré par les Kanak comme leur lieu de naissance, et régulièrement survolé par ces petits mammifères notamment durant les périodes cycloniques). F. Brescia avait identifié que l'approche ethnoécologique que j'avais mise en œuvre dans les projets de recherche conduits sur le territoire et dans d'autres régions auparavant pouvait apporter des réponses plus rapidement et plus complètement que l'approche " purement » écologique. Des années d'observations sur plusieurs générations par des chasseurs, des pêcheurs ou tout praticien de l'environnement ayant des interactions régulières et parfois quotidiennes avec son environnement forestier, côtier, marin sont d'une richesse inestimable, et notamment pour les chercheurs des sciences naturelles. J'en étais persuadée mais cette sollicitation a été pour moi la première qui a permis que notre approche soit pensée en amont d'une étude écologique. Cela me permet aujourd'hui d'écrire que l'ethnoécologie peut - et doit - jouer un rôle moteur dans la réalisation de recherches interdisciplinaires touchant aux relations entre sociétés et natures. Une partie des outils utilisés durant le terrain ethnographique a été coconstruite afin de rendre compte le plus complètement et finement possible des savoirs écologiques locaux sur les roussettes et leur environnement. Puis une réflexion a été engagée d'une part sur les enjeux épistémologiques qui émergeaient de notre collaboration; et d'autre part sur le statut d'une connaissance produite par la combinaison de savoirs locaux et de données naturalistes ${ }^{2}$. Enfin, la collectivité provinciale s'est saisie de nos résultats croisés pour travailler leur code de l'environnement.

Une autre expérience de recherche interdisciplinaire marquant particulièrement ces dernières années est celle du programme de recherche ESPAM, Espèces emblématiques, acceptation sociale et aires marines protégées, financé par la Fondation de France. Cette recherche a émergé d'expériences individuelles et collectives en Nouvelle-Calédonie et à La Réunion. Constatant les difficultés rencontrées par les agents des collectivités publiques en charge de la gestion des territoires marins pour s'approprier complètement les résultats des recherches de sciences humaines et sociales et s'y référer afin de convaincre leurs hiérarchies dans l'ajustement des politiques, nous avons proposé d'envisager des manières innovantes pour collecter, analyser et restituer nos travaux. Notre question principale était la suivante: comment appréhender de manière sensible les savoirs, les valeurs sociales et culturelles accordées aux animaux et aux territoires marins en Nouvelle-Calédonie et à La Réunion et les rendre utiles et compréhensibles par ceux qui construisent les politiques et les font appliquer? De nouveau, mon ancrage dans les ethnosciences au sein d'un chemin riche en diversité méthodologique et théorique m'a facilité l'adoption d'un certain éclectisme théorique cher à Olivier de Sardan pour collecter et analyser nos données empiriques (Olivier de Sardan 2008) en compagnie de mes collègues d'autres disciplines. Pour cette recherche, la biologie, les mathématiques, la science de données, la géographie et l'anthropologie ont apporté leurs pierres respectives pour allier des approches qualitatives et quantitatives afin d'appréhender les valeurs, les représentations et les savoirs locaux, et de produire des outils de dialogue plus accessibles aux divers acteurs territoriaux (Figure 4). Les différentes dimensions 
marquant les liens entre les humains et les animaux marins ont été caractérisées. Et audelà des valorisations classiques de nos résultats via des restitutions orales et des publications scientifiques, ces dimensions ont été traduites sous forme de visuels qui ont déjà convaincu plusieurs de nos partenaires (Sabinot et al. 2021).

Figure 4 : La diversité des espèces marines emblématiques en Nouvelle-Calédonie

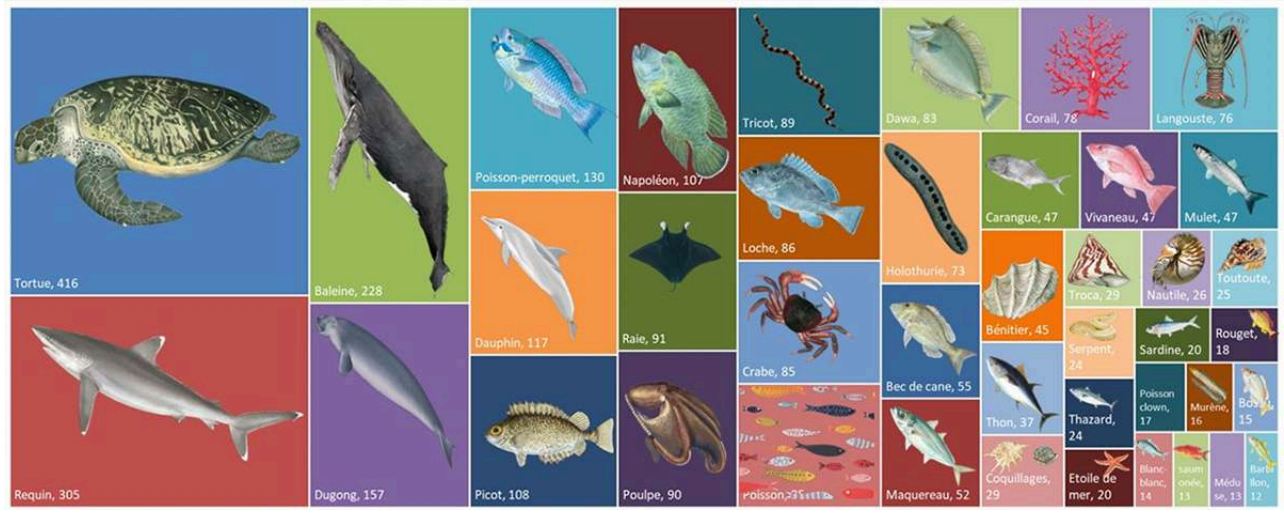

L'illustration représente les espèces citées au moins dix fois en Nouvelle-Calédonie (496 personnes interrogées). Les surfaces sont proportionnelles à la fréquence des citations.

Illustrations d'animaux marins reproduites avec l'aimable autorisation de la Communauté du Pacifique (CPS). Hazel Adams, Youngmi Choi, Les Hata et Rachel O'Shea, (c) CPS

\section{De la connaissance à la reconnaissance des savoirs locaux, des visions du monde et de l'éthique qu'ils portent}

Ces expériences ont permis de contribuer à la connaissance et la reconnaissance des valeurs et des savoirs locaux et de rappeler au monde scientifique et au monde politique à qui les résultats ont été présentés que les savoirs des habitants produits par leur expérience de l'environnement « sont également des savoir-faire, un mode de vie, une représentation du monde et une éthique » (Roué 2012). L'ethnoécologie invite ainsi autant à comprendre et rendre compte de la diversité des savoirs écologiques qu'à appréhender comment ces savoirs influencent la manière d'être au monde des individus et s'inscrivent dans une éthique singulière. Les savoirs écologiques sont en effet le reflet de multiples modalités de relations aux autres, humains et non-humains. Avec Nicolas Lescureux, également docteur du MNHN et désormais ethnoécologue au CNRS, nous avons rappelé dans un ouvrage traitant de la coviabilité que l'ethnoécologie offre de "décrire les manières de catégoriser, de classifier, d'organiser les connaissances en un système de savoirs" mais aussi "de construire une analyse comparative et symétrique entre diverses sociétés, y inclus les nôtres" (Sabinot \& Lescureux 2019).

Cette perspective comparative doit permettre de rendre compte qu'il n'y a pas une seule manière de penser les relations aux autres, qu'ils soient humains ou non humains. Elle doit aussi offrir au plus grand nombre la possibilité de s'emparer de ces conclusions pour construire une diversité de transitions qui assureront le maintien des liens entre les êtres qui forment nos vies, nos territoires. J'adopte cette perspective comparative aussi souvent que possible en Nouvelle-Calédonie, territoire marqué par une 
décolonisation négociée singulière toujours en cours ${ }^{3}$ et par une population plurielle et métissée; dans les autres pays et territoires d'Océanie où la diversité des manières de voir le monde est entre autres portée par une diversité linguistique exceptionnelle ${ }^{4}$; et plus largement sur les territoires insulaires des trois océans où nous nous efforçons avec les partenaires des territoires, de décrire les transformations dans les savoirs et pratiques des pêcheurs et des sociétés insulaires et côtières au rythme des changements sociaux et environnementaux ${ }^{5}$. Le nous que j'emploie ici est englobant et inclut en particulier mon collègue Gilbert David ancré dans une géographie culturelle qui porte des ambitions identiques à celles portées par l'approche anthropologique que je pratique. Il est devenu depuis 2012 un compagnon de réflexion fondamental et inspirant confortant le rôle de l'ethnoécologie et de l'anthropologie en général dans les projets et les enseignements que nous faisons vivre.

En dehors du monde académique, les premiers qui nourrissent ma compréhension, ce sont les pêcheurs, les pêcheuses et leurs familles. Ils se confient, m'accueillent dans leurs parties de pêche et dans les divers moments qui rythment leur vie. J'étais « la fille qui écrit » lorsque je réalisais mon travail de terrain de thèse. J'étais aussi au Gabon, au Mexique ou plus récemment au Vanuatu « celle qui connaît comment se fait la pêche d'ailleurs », qui « peut te montrer ». La richesse des savoirs qui m'ont été transmis, sauf si ceux-ci devaient rester confidentiels, je pouvais à mon tour les partager, et rapporter à ces gens qui ne se rencontreront probablement pas comment d'autres font, comment ils s'organisent, comment ils construisent et font évoluer leurs relations aux autres, humains et non-humains. Que des manières si différentes existent pour pêcher le poulpe à travers la planète fascinent les praticiens de la mer. Cela ne remet pas en cause leur manière de pêcher mais ils me disent être curieux de connaître, de voir et de se rendre compte de cette diversité. A l'inverse, mes interlocuteurs sont aussi ravis d'observer des similitudes entre leurs manières de voir ici sur une petite île du Pacifique et celles des côtes guyanaises, haïtiennes ou mozambicaines, tant dans les représentations du monde invisible que dans les pratiques de capture mises en œuvre pour cibler des espèces de poissons ou de crustacés. Cette envie d'en apprendre sur les autres a aussi parfois ravivé chez les personnes avec qui j'échangeais, l'envie de transmettre au sein de leur cercle familial ou amical proche, car ils constataient comme dans de nombreux autres lieux un étiolement dans la maîtrise de la langue, de la connaissance de ce qui les entoure et de certains savoir-faire. Pour toutes ces raisons, ce que nous relatons dans nos écrits mérite d'être chaque fois que c'est possible transmis oralement à ceux qui nous nourrissent.

Et depuis quelques mois, nous expérimentons par la création artistique numérique une nouvelle voie pour partager le cœur de nos recherches et de nos ambitions. Dans le cadre d'une convention IUCN-IRD, l'artiste calédonien Nicolas Molé travaille avec nous sur une animation numérique "Savons-nous toujours écouter la nature ?", visant à valoriser les savoirs et les savoir-faire locaux pertinents pour la résilience des sociétés et le maintien d'une biodiversité en santé, et à rappeler le rôle des sciences humaines et sociales aux côtés des autres sciences pour penser les politiques de gestion de l'environnement. (https://youtu.be/g-HbZpgzaQw). 


\section{L'ethnoécologie pour appréhender les dynamiques des sociétés et rendre compte des résiliences éprouvées}

17 Les sciences humaines et sociales ont un rôle majeur à jouer pour éloigner nos décideurs de visions trop technocratiques qui mettraient au centre de leurs actions des questions techniques et économiques. L'ethnoécologie peut aider à placer au centre du débat les enjeux sociaux, culturels et politiques au même titre que les enjeux économiques, et espérer en conséquence une meilleure prise en compte de la diversité des modalités de relations aux autres qui sont autant de chemins pour penser les résiliences des sociétés et des territoires.

18 L'ethnoécologie analyse les liens entre les êtres qu'ils soient humains ou non-humains. Elle cherche aussi à décrire le lien entre le savoir et l'action (Toledo 1992), notamment en rapport avec la gestion du territoire et des ressources. Le concept de TEK (Traditional Ecological/Environmental Knowledge) et ses cousins IK (Indigenous Knowledge), TKW (Traditional Knowledge and Wisdom) et LEK (Local Ecological/Environmental Knowledge) contiennent résolument cette notion. Dans ces considérations, il importe de ne pas réduire les savoirs locaux à un caractère « traditionnel » souvent présupposé figé (pour qui ? depuis quand ? etc.). Il est essentiel de défendre sans cesse cette vision dynamique des savoirs auprès des chercheurs de toute discipline, des étudiants à qui nous enseignons et de tout citoyen intéressé, en particulier ceux impliqués dans les collectivités qui œuvrent pour la société et la gouvernance des interactions entre les humains et les non-humains. Ces non humains sont plus fréquemment institutionnellement nommés " ressource ", «nature » ou " environnement » alors que ces termes n'ont bien souvent pas d'équivalents dans les langues vernaculaires. En Océanie par exemple, ces traductions ne sont pas possibles : il n'y a pas de distinction entre l'homme et la nature mais une diversité de liens entre humains et entre humains et non-humains.

19 L'entrée par les savoirs écologiques est particulièrement pertinente pour appréhender les transformations, les moments d'adaptation et de résilience qui caractérisent les sociétés, notamment dans le Pacifique où les populations se sont constituées des cultures du risque (David 2005) pour faire face à des phénomènes extrêmes récurrents tels les cyclones, les tsunamis ou les sécheresses. Un nouveau programme de recherche combinant les sciences du climat et l'ethnoécologie démarre cette année en partenariat avec l'Agence française de développement. Nommé CLIPSSA pour CLImat du Pacifique, Savoirs locaux et Stratégies d'Adaptation, il combinera les sciences du climat, la géographie et l'ethnoécologie pour produire des données inédites sur le climat futur du Pacifique et sur les cultures $d u$ risque variées construites par les populations des îles du Vanuatu, de la Nouvelle-Calédonie, de Wallis et Futuna et de la Polynésie française. Il permettra de connaître, à des résolutions fines, certaines caractéristiques clés pour le climat futur, comme les précipitations moyennes, les températures et évènements de précipitations extrêmes ou les cyclones. Seront en parallèle analysés les ressentis et vécus des populations face aux impacts de ces phénomènes extrêmes, ainsi que l'évolution des savoirs locaux mobilisés pour y faire face. Enfin, seront étudiées les voies envisageables pour favoriser l'intégration dans les politiques d'adaptation de la diversité des savoirs scientifiques et locaux existants ou coproduits.

Un des défis auquel s'attachera notre recherche sera d'identifier les savoirs et savoirfaire écologiques locaux qui peuvent favoriser la résilience des populations face au 
changement climatique, et d'analyser la diversité des lieux et des modalités de transmission de savoirs existant. Nous identifierons entre autres dans chaque archipel quels sont les éléments qui induisent différents types de réaction face aux changements rencontrés; quels sont les motifs qui conduisent parfois à la remobilisation de savoirs et savoir-faire anciens et pourquoi certains canaux de transmission y sont favorables et d'autres moins. Enfin, nous observerons si ces dynamiques entraînent des transformations dans d'autres savoirs, savoir-faire ou savoir-être.

21 Combiner les résultats émanant de notre approche ethnoécologique à ceux émanant des sciences du climat au fur et à mesure de leurs productions permettra, d'une part d'orienter les entretiens semi-directifs pour discuter des situations climatiques qui ont le plus de probabilité de survenir et, d'autre part, d'orienter les modélisations climatiques de nos collègues sur des lieux ou des enjeux plus prégnants du point de vue de nos interlocuteurs.

Ce programme de recherche interdisciplinaire sera suivi par un appui financier et humain de l'AFD $^{6}$ auprès des gouvernements concernés afin de leur permettre de penser des stratégies et des plans d'adaptations incluant les résultats de recherche que l'équipe aura produit. La recherche fondamentale menée conjointement aura ainsi plus de chances de traverser le monde non-académique dans des délais raisonnables pour se retrouver au cœur de nouvelles politiques.

\section{L'ethnoécologie pour construire des passerelles avec le monde non-académique et favoriser l'inclusion des savoirs locaux dans les politiques}

Comprendre les dynamiques sociétales et territoriales vécues par les sociétés côtières et insulaires et saisir les processus de transmission des savoirs comme les articulations qui se jouent entre les différents types de savoirs, au sein des catégories locales (LEK) comme institutionnelles - savoir local/savoir normé - sont les deux ambitions fondamentales que visent nos recherches futures portées par l'ethnoécologie. Un objectif plus opérationnel qui nous guide est d'œuvrer pour la reconnaissance de la diversité de ces savoirs, de ces manières de voir et d'organiser le monde. Cette diversité mérite en effet d'être au cœur des préoccupations sociétales et en particulier celles prétendant gérer l'environnement ou favoriser l'adaptation des sociétés aux impacts du changement climatique.

24 Afin de renforcer l'interface science-politique dans les problématiques de gestion de l'environnement, un organe intergouvernemental indépendant a été créée en 2012. Il s'agit de l'IPBES, plateforme intergouvernementale scientifique et politique sur la biodiversité et les services écosystémiques. Je l'ai rejointe en 2019 en tant qu'ethnoécologue pour contribuer à l'écriture d'un rapport de cadrage dont nous discuterons plus loin et à diverses évaluations. L'IPBES a pour mission de fournir aux décideurs des évaluations scientifiques objectives de l'état des connaissances sur la biodiversité et des outils et méthodes existant pour protéger et utiliser de manière durable les ressources naturelles vitales. L'objectif in fine et de favoriser la conservation et l'utilisation durable de la biodiversité, le bien-être de l'humanité à long terme et le développement durable. Des centaines de chercheurs provenant de plusieurs dizaines des pays différents contribuent à ces rapports. Après avoir entre autres travaillé sur 
quatre évaluations régionales de la biodiversité, l'IPBES a produit en 2019 le premier rapport d'évaluation mondial sur la biodiversité et les services écosystémiques. Il s'appuie sur près de 15000 références et constitue la toute première évaluation qui examine systématiquement et intègre les savoirs, problématiques et priorités autochtones et locaux ${ }^{7}$. Depuis, cette plateforme s'est engagée dans la production d'évaluations ciblant des thématiques transversales à tous les continents au sein desquelles les ethnosciences ont un rôle majeur à jouer. Deux rapports de cadrage ont entre autres été récemment rédigés, chacun par une quarantaine d'experts provenant de différents pays et champs disciplinaires. L'un pose les bases pour la rédaction de l'évaluation Nexus qui portera sur les liens entre la biodiversité, le changement climatique, les processus d'adaptation et d'atténuation. Il s'intéressera aux articulations entre les secteurs de l'énergie, de l'eau, de l'alimentation et de la santé. L'autre pose les bases de l'évaluation Changements transformateurs, ces derniers étant compris comme une réorganisation fondamentale, en profondeur, "à l'échelle de l'ensemble des facteurs technologiques, économiques et sociaux, y compris des paradigmes, des objectifs et des valeurs " (IPBES 2021), visant à la conservation et à l'utilisation durable de la biodiversité, au bien-être humain à long terme et au développement durable ${ }^{8}$. Le groupe d'experts sera entre autres en charge d'évaluer, en s'appuyant sur la littérature existante, scientifique comme grise, les options pratiques et les actions concrètes permettant de réaliser des visions, des scénarios et des trajectoires conduisant à un monde durable, et de proposer de manières de définir et de mesurer les avancées vers des changements transformateurs.

Plus encore que dans les évaluations régionales, les chercheurs des sciences humaines et sociales et notamment les ethnoécologues et les " experts des savoirs locaux", pour reprendre les termes de l'Ipbes, sont invités à y contribuer. Il s'agira pour nous de synthétiser où en sont les recherches sur ces thématiques, de rendre compte de la diversité d'une part des manières de voir les relations entre humains et non-humains sur la planète, et d'autre part des dynamiques d'ajustements et de transformations de ces relations qui existent de par le monde. Identifier les initiatives, les alternatives à la façon de voir l'environnement qui domine encore le monde en séparant l'homme de la nature, sera un des défis que devront relever nos contributions d'ethnoécologues.

\section{Pour conclure}

Comme nous y ont invités les éditeurs de ce numéro anniversaire de la Revue d'ethnoécologie, cet article a tenté de partager une expérience de l'ethnoécologie parmi d'autres dans un parcours d'une lycéenne qui ne connaissait pas l'existence de la discipline en 1998, qui cherchait à contribuer à comprendre l'évolution du rapport des hommes à leur environnement mais aussi à donner aux autres l'envie de comprendre, d'apprendre, de transmettre, interroger. Je suis devenue docteure en ethnoécologie au Muséum national d'Histoire naturelle de Paris en 2008 puis chercheure à l'Institut de Recherche pour le Développement trois années après. C'est l'étude des liens entre les sociétés et la mer et de la transmission des savoirs qui me portait. Les opportunités qui m'ont été données de travailler auprès de sociétés vivant de la mer et pensant leurs relations au monde différemment les unes des autres, et bien souvent différemment de la pensée occidentale dominante concevant l'homme et la nature comme dichotomique, 
m'ont conduites sur les littoraux et îles de l'ouest et de l'est de l'Atlantique, puis dans l'Indopacifique.

Aux questions posées par le Comité de rédaction, lorsqu'il m'a sollicitée, j'ai tenté d'apporter un regard, de partager quelques moments de recherches et d'interactions avec d'autres chercheurs ou avec des citoyens et acteurs des collectivités publiques. Quelques questions méritent encore courtes réponses.

Y a-t-il une transmission entre les générations, réappropriation de l'ethnoécologie? Oui ! Et ce champ disciplinaire qui n'était pas enseigné à l'Université de la Nouvelle-Calédonie et au Vanuatu l'est désormais et amène plusieurs étudiants à s'engager dans cette approche au niveau Master et Doctorat. ou bien celles faisant l'objet des recherches? Développer des approches ancrées dans l'ethnoécologie, a permis de construire des ponts entre les disciplines autour d'enjeux de recherche et de développement concrets dont se saisissent aujourd'hui des associations, et des acteurs des collectivités, mais aussi des individualités qui se sont motivées ou remotivées pour contribuer à la collecte des savoirs dans leur entourage, voire qui ont réactivé leur transmission qui s'était nettement étiolée.

Quel est le futur de ces domaines intellectuels? L'ethnoécologie gagnera à s'enrichir aux côtés des autres disciplines comme elle l'a fait depuis un siècle. Sur tous les lieux où ce champ disciplinaire sera mobilisé, il devra être ouvert à être bousculé pour sans cesse se renouveler afin de répondre aux défis de compréhension et d'action, de transition qui se dessinent. Au-delà de contribuer à la reconnaissance des savoirs locaux et des manières singulières de concevoir les rapports entre sociétés et natures, «nourrir les réflexions sur l'apprentissage, la formation, la scolarisation comme sur le développement territorial» (David et al. 2018: 159) gagnera à être au cœur de nos travaux futurs, comme nous l'avons rappelé avec G. David et E. Hnawia dans un article discutant du rôle de la recherche scientifique dans la réduction de la décolonisation et des inégalités sociales.

\section{BIBLIOGRAPHIE}

David G. 2005 - Spatialité et temporalité océanes, Recherches sur les îles et les rivages de l'Océanie et de la partie occidentale de l'océan Indien. Mémoire d'habilitation à diriger des recherches, Paris, Université Paris IV-Sorbonne.

David G., Hnawia E. \& Sabinot C. 2018 - Inégalités sociales et décolonisation : le rôle de la recherche scientifique. In : Faberon F., Wamytan L., Lauouvea I. \& Paturet A. (Ed.), Inégalités sociales et décolonisation : les rééquilibrages de la Nouvelle-Calédonie. Nouméa ; Clermont-Ferrand ; Toulouse, Editions L'Épitoge-Lextenso : 145-159.

Doyon S. \& Sabinot C. 2015 - Anthropologie des espaces côtiers et de la conservation environnementale. Pêche, sel et flamants roses dans les Réserves de biosphère yucatèques au Mexique. Presses de l'Université Laval. 
Friedberg C. 2005 - Ethnoscience et autres ethno «machins » aujourd'hui. Journal de la Société des Océanistes 120-121:27-30.

IPBES 2021 - Rapport de cadrage pour une évaluation thématique des causes profondes de l'érosion de la biodiversité, des déterminants des changements transformateurs et des solutions pour réaliser la Vision 2050 pour la biodiversité.

ISEE 2020 - Recensement de la population 2019, Nouvelle-Calédonie. Synthèse nº 45 .

Olivier De Sardan J.-P. 2008 - La rigueur du qualitatif : les contraintes empiriques de l'interprétation socio-anthropologique. Louvain-La-Neuve, Academia-Bruylant.

Roue M. 2012 - Histoire et épistémologie des savoirs locaux et autochtones. Revue d'ethnoécologie (1). doi.org/10.4000/ethnoecologie.813

Sabinot C. 2008 - Dynamique des savoirs et des savoir-faire dans un contexte pluriculturel. Étude comparative des activités littorales au Gabon. Thèse, Muséum National d'Histoire Naturelle, Paris.

Sabinot C., Delebecque S., Cillaurren E., Fossier C., Pennober G., Rodary E. \& David G. 2021 Espèces emblématiques et gestion de la mer, regards pluridisciplinaires en sciences sociales et nouvelles approches méthodologiques dans l'outre-mer indo-pacifique. Norois. Environnement, aménagement, société 259-260 : 181-203.

Sabinot C. \& Lescureux N. 2019 - The Local ecological knowledge and the viability of the relations with the environment. In : Barrière O., Behnassi M., David G., Douzal V., Fargette M., Libourel T., Loireau M., Pascal L., Prost C., Ravena-Cañete V., Seyler F. \& Morand S. (Ed.), Coviability of Social and Ecological Systems: Reconnecting Mankind to the Biosphere in an Era of Global Change, Vol.1: The Foundations of a New Paradigm. Cham, Springer International Publishing : 211-222.

Toledo V.M., 1992 - What is ethnoecology? Origins, scope and implications of a rising discipline. Etnoecologica, $1: 5-21$.

\section{NOTES}

1. IPBES, Plateforme intergouvernementale scientifique et politique sur la biodiversité et les services écosystémiques

2. Des données naturalistes ont également été collectées durant cette étude et des suivis des populations de roussettes ont été mis en place.

3. Le troisième et dernier référendum pour l'autodétermination prévu par les Accords de Nouméa a eu lieu le 12 décembre 2021.

4. Ainsi, le Vanuatu par exemple possède la plus forte densité linguistique du monde avec 108 langues vernaculaires distinctes pour 272000 habitants et 81 îles pour une superficie totale de $12189 \mathrm{~km}^{2}$ de surface émergée.

5. Recherches menées en Haïti, au Mexique, dans les Guyanes et au Brésil, au Sénégal, au Gabon et en Guinée Conakry pour ce qui est de l'Océan atlantique ; en Afrique de l'Est, à Mayotte, aux Comores, à Madagascar et à La Réunion pour l'Océan indien ; en Nouvelle-Calédonie, en Polynésie française, au Vanuatu et aux îles Fidji pour ce qui est de l'Océan pacifique.

6. AFD Agence Française du Développement

7. Pour de plus amples informations, consulter le site de l'Ipbes (https://ipbes.net/) et de la Fondation pour la recherche sur la biodiversité (FRB) (https://www.fondationbiodiversite.fr/) qui accueille le secrétariat scientifique du comité français pour l'Ipbes.

8. Ib. 


\section{RÉSUMÉS}

Nos pratiques actuelles de l'ethnoécologie dans le Pacifique se sont résolument construites depuis les ethnosciences nées au Museum national d'histoire naturelle de Paris et marquées par plusieurs grands acteurs de la discipline aujourd'hui disparus. Déployés, éprouvés, osés, parfois bousculés sur les côtes et les îles des océans atlantiques, indiens et pacifiques, les concepts et les outils portés par ces ethnosciences ont nourri nos recherches. Les ethnosciences se sont montrées être un champ de compréhension du monde essentiel pour reconnaitre et faire reconnaître les savoirs des praticiens de l'environnement, ceux qui ont des interactions régulières, voire quotidiennes avec les non-humains. Elles sont devenues durant mon expérience de chercheure sur les trois océans sujets de débats avec les pêcheurs et autres métiers de la terre et de la mer comme avec les acteurs de la conservation et du développement œuvrant dans les organisations non-gouvernementales ou dans les collectivités publiques. Avec le temps, les savoirs et manières de voir le monde sont révélés par ce champ de recherche et ont peu à peu traversé les frontières académiques et non-académiques ; ils sont aujourd'hui fréquemment au cœur de programmes de recherche réellement interdisciplinaires, et même au cœur des nouveaux rapports d'évaluations de l'organisation intergouvernementale qu'est l'IPBES ${ }^{1}$. Afin de renforcer l'interface science et politique dans le domaine de la biodiversité et des services écosystémiques en vue de la conservation et de l'utilisation durable de la biodiversité, du bienêtre de l'humanité à long terme et du développement durable, l'IPBES, après avoir travaillé sur des évaluations régionales de la biodiversité, s'est engagée dans la production d'évaluations ciblant des thématiques transversales à tous les continents au sein desquelles nous verrons que les ethnosciences ont un rôle majeur à jouer.

Our current practices of ethnoecology in the Pacific have been resolutely built up on the ethnosciences born at the Muséum national d'Histoire naturelle in Paris and marked by several major figures of the discipline who have now passed away. Deployed, tested, bold, and sometimes shaken up on the coasts and on the islands of the Atlantic, Indian and Pacific oceans, the concepts and tools used by these ethnosciences have nourished our research. Ethnosciences have proven to be an essential field to understand the world and acknowledge the expertise of environmental practitioners; those who have regular, if not daily, interactions with non-humans. During my experience as a researcher on the three oceans, ethnosciences became the subject of debate with fishers and other people working on the land and sea, as well as with conservation and development stakeholders working in non-governmental organizations or public communities. Over time, the knowledge and ways of looking at the world revealed by this field of research have gradually crossed academic and non-academic boundaries and are now frequently at the heart of truly interdisciplinary research programs, and even at the heart of the new assessment reports of the IPBES, intergovernmental organization. In order to strengthen the science-policy interface in the field of biodiversity and ecosystem services for the conservation and sustainable use of biodiversity, long-term human well-being and sustainable development, IPBES, after having worked on regional assessments of biodiversity, is now engaged in the production of assessments targeting cross-cutting themes in which we will see that the ethnosciences have a major role to play. 
INDEX

Mots-clés : ethnosciences, Pacifique, interfacescience-société, engagement, parcours de chercheur, Nouvelle-Calédonie

Keywords : ethnosciences, Pacific, New-Caledonia, engagement, researchers' path, interdisciplinarity

\section{AUTEUR}

\section{CATHERINE SABINOT}

Ethnoécologue et anthropologue, UMR 228 ESPACE-DEV, IRD, Université de Montpellier, Université des Antilles, Université de Guyane, Université de la Réunion, Université de la Nouvelle-Calédonie - Centre IRD Anse Vata - BPA5 98848 Nouméa Cedex - Nouvelle-Calédonie 